Assessing the Effects of Data Compression in Simulations Using Physically Motivated Metrics

D. E. Laney, S. Langer, C. Weber, P. Lindstrom, A. Wegener

August 12, 2013

Supercomputing

Denver, CO, United States

November 17, 2013 through November 22, 2013 
This document was prepared as an account of work sponsored by an agency of the United States government. Neither the United States government nor Lawrence Livermore National Security, LLC, nor any of their employees makes any warranty, expressed or implied, or assumes any legal liability or responsibility for the accuracy, completeness, or usefulness of any information, apparatus, product, or process disclosed, or represents that its use would not infringe privately owned rights. Reference herein to any specific commercial product, process, or service by trade name, trademark, manufacturer, or otherwise does not necessarily constitute or imply its endorsement, recommendation, or favoring by the United States government or Lawrence Livermore National Security, LLC. The views and opinions of authors expressed herein do not necessarily state or reflect those of the United States government or Lawrence Livermore National Security, LLC, and shall not be used for advertising or product endorsement purposes. 


\section{Assessing the Effects of Data Compression in Simulations Using Physically Motivated Metrics}

\author{
Daniel Laney \\ Lawrence Livermore Lab \\ dlaney@llnl.gov \\ Peter Lindstrom \\ Lawrence Livermore Lab \\ pl@IInl.gov
}

\author{
Steven Langer \\ Lawrence Livermore Lab \\ langer@llnl.gov
}

\author{
Christopher Weber \\ Lawrence Livermore Lab \\ weber30@ilnl.gov
}

\author{
Al Wegener \\ Samplify \\ awegener@samplify.com
}

\begin{abstract}
This paper examines whether lossy compression can be used effectively in physics simulations as a possible strategy to combat the expected data-movement bottleneck in future high performance computing architectures. This paper shows that, in a number of cases, compression levels of 3-5X can be applied without causing a significant change in the physical quantities that are of most interest for each simulation.

Rather than applying classical error metrics from signal processing, we utilize physics-based metrics appropriate for each code to evaluate the impact of compression. We evaluate simulations run with three different codes: a Lagrangian shock-hydrodynamics code, an Eulerian higher-order hydrodynamics turbulence modeling code, and an Eulerian coupled laser-plasma interaction code. We apply compression to relevant quantities after each time-step to approximate the effects of tightly coupled compression and also study the compression rates to estimate memory and disk-bandwidth reduction. We find that the error characteristics of compression algorithms must be carefully considered in the context of the underlying physics being modeled.
\end{abstract}

\section{Keywords}

data compression, high performance computing

\section{INTRODUCTION}

The computing power of large systems is increasing faster than their memory and disk bandwidth. A number of reports have indicated that this will lead to serious difficulties in effectively exploiting proposed Exascale $\left(10^{18}\right.$ floating point operations per second) systems. In particular, a large number of applications will be limited by memory bandwidth rather than processor performance [1,2]. Applications with large memory footprints may be unable to write a checkpoint to disk in a time short compared to the mean time between application interrupt (MTBAI). The memory

Permission to make digital or hard copies of all or part of this work for personal or classroom use is granted without fee provided that copies are not made or distributed for profit or commercial advantage and that copies bear this notice and the full citation on the first page. To copy otherwise, to republish, to post on servers or to redistribute to lists, requires prior specific permission and/or a fee. Request permissions from Permissions@ acm.org. SC13 November 17-21, 2013, Denver, CO, USA

Copyright 2013 ACM 978-1-4503-2378-9/13/11 ...\$15.00.

http://dx.doi.org/10.1145/2503210.2503283 per core will also drop, forcing some strong scaling relative to current systems. It will be difficult to increase either disk bandwidth, memory bandwidth, or memory capacity above projections due to power constraints.

This paper examines whether compression can be used effectively in physics simulations to mitigate these effects by reducing data size and therefore bandwidth requirements. In effect, compression provides the opportunity to trade off additional computation in exchange for reduced bandwidth. Lossless compression ratios are modest for the codes we consider and thus will only have modest impact on memory bandwidth requirements at Exascale. For example, data arrays from LLNL's pF3D code can only be losslessly compressed by a factor of $1.17 \mathrm{X}$ by gzip, and $1.29 \mathrm{X}$ by fpzip, one of the floating point compression schemes used in this paper. While many physics simulations start out with large uniform (constant) regions, lossless compression rates decline quickly once a simulation starts running as, in many cases, the low order significand of state variables rapidly take on a semi-random character. Therefore, to have a significant impact on the memory or disk bandwidth requirements of simulations, lossy compression methods are necessary.

Lossy compression is most frequently used for photographs and for videos where key-frames periodically reset the error to zero. The physics codes we consider contain run timevarying dependencies whose errors can potentially accumulate. If the compression errors at one time step are strongly correlated with errors at the next time step, the cumulative effect might grow more rapidly than if they are uncorrelated. A compression algorithm should be judged both on the level of compression it achieves and the characteristics of the differences it generates.

We are interested in two possible uses of compression: "disk compression" and "memory compression". In "disk compression," arrays are compressed before they are written to disk; in "memory compression," arrays are compressed while stored in off-chip memory and decompressed when they are loaded into on-chip cache for computation.

Disk compression can have a positive impact on performance today. For example, the bandwidth per core to the parallel file system on Cielo, a Cray XE-6 at Los Alamos National Laboratory, is roughly $1 \mathrm{MB} / \mathrm{s}$ for $\mathrm{pF} 3 \mathrm{D}$ when $1 \mathrm{k}$ to $64 \mathrm{k}$ cores write simultaneously. Lossy compression algorithms have a throughput of roughly $200 \mathrm{MB} / \mathrm{s}$, so the time spent compressing data would be small compared to the time spent writing it to disk. The time spent writing to disk would be reduced by roughly the compression ratio. 
Memory compression will not help performance on current multi-core systems because memory bandwidth per core (roughly $8.6 \mathrm{~GB} / \mathrm{s}$ on an Intel Sandy Bridge) is significantly higher than software compression rates. The memory bandwidth per core is likely to drop on future systems, which will make memory compression more viable. An interesting possibility is to put compression in on-chip logic. The APAX compression algorithm can achieve throughputs of 2.5 to $8 \mathrm{~GB} / \mathrm{s}$ while using between 0.1 and $0.4 \mathrm{~mm}^{2}$ in $28 \mathrm{~mm}$ CMOS and increasing the power consumption of a chip by less than one percent. This rate is close enough to the memory bandwidth per core that memory compression might increase overall application performance.

Another benefit of memory compression is that it would allow our codes to fit more zones in the available memory. In particular, compression would allow us to fit more zones in the fast GDDR memory on current Nvidia and Intel accelerator boards or the stacked memory on proposed future systems while reduce accesses to the slower DDR memory.

The main challenge when applying lossy compression is assessing its effect on simulation accuracy. Depending on the chosen compression ratio, lossy compression will result in differences larger than arthmetic roundoff. That is not a show-stopper - many decisions made in setting up a simulation also change the answer. The scientist running a code picks a zone size based on a tradeoff between more accurate answers and the computational cost of the simulation. Computational scientists usually make these choices based on the desired accuracy of various integral physical quantities, not by bounding the expected zone-by-zone differences between two proposed simulations.

We propose using the same integral physical quantities to assess the impact of compression. In our approach, the code developer runs test problems with and without compression and evaluates key physical quantities. The results can be used to select the best compression algorithm and the proper compression level. An important consideration is that memory compression has a larger impact on the accuracy of a simulation than disk compression because it occurs hundreds of times more frequently than checkpointing. Our approach is to apply compression at the end of every cycle, in order to simulate the effects of memory compression. Our results also provide an upper bound on how much loss could be tolerated in checkpoints. Where applicable we also investigate lossy compression at checkpoint frequencies to verify this. This paper shows that, in the simulation codes we examined, 3-5X compression ratios can be applied without causing a significant change in the physical quantities that are of most interest for the simulation.

We evaluate the impact of compression on three different simulation codes: LULESH, a shock hydrodynamics miniapp; Miranda, a hydrodynamics code for large turbulence simulations; and $\mathrm{pF} 3 \mathrm{D}$, which simulates the interaction of a high intensity laser and a plasma (ionized gas). This paper is organized into three sections. Each section begins with a high-level discussion that should be accessible to a general HPC audience. The rest of the section provides details that may only be of interest to experts. The final section summarizes our results and indicates topics for future research.

\section{PRIOR ART}

Numerical compression has an exceedingly rich history dating back to the 1960s. However, the compression re- search community has understandably focused on compression of consumer speech, audio, images, and video, given the ubiquity of such media. Media compression techniques are inappropriate for a universal numerical encoder because the quality metrics for media compression are determined by limitations of human hearing and vision, not by the accuracy requirements of numerical computations. Compressive sensing (CS) $[3,4]$ is targeted towards real-time sensor systems but requires significant back-end complexity to unwind the analog front end's random sampling. Waveform coders $[5,6]$ encode integer samples but can lack flexibility. Lossless compression of scientific data [7-10] comes closest to a universal numerical encoder but typically achieves less than a $2 \mathrm{X}$ compression ratio on floats and even lower ratios on doubles. Furthermore, most such algorithms do not support lossy compression.

Lossy compression for scientific and medical data has historically been applied to data sets to reduce their size for post-processing and visualization. Muraki et al. [11] pioneered the application of wavelet transforms to volumetric data for visualization applications, thresholding wavelet coefficients to obtain approximate representations and applying non-orthogonal wavelets to medical images [12]. More recently, Woodring et al. [13] explored the use of wavelet compression in JPEG2000 to enable scientists to trade off data quality for reduced data size. Their approach targeted reduced disk $\mathrm{I} / \mathrm{O}$ time in visualization applications and faster transfers of data from remote systems to local assets.

ISABELA [14] has been proposed as a data reduction approach for HPC applications, particularly targeted at data deemed effectively incompressible due to randomness or noise. The data is partitioned into blocks and the data in each block is sorted by value. Splines are fitted to the resulting monotonic data using a relatively small number of knots. Temporal patterns are leveraged to further increase compression ratios. The approach has several advantages for disk-compression, particuarly since the method does not require global communication and can be run in-situ. Lehmann et al. [15] present a modified version of ISABELA suited to compressing flow simulations in porous media.

The simulations presented in this paper operate repeatedly on data that has been previously compressed, leading to the possibility of amplification of compression-induced differences. In the next section we discuss compression algorithms in this context, and motivate our choice to use predictive coders in our experiments.

\section{COMPRESSION ALGORITHMS}

In most physics simulations, the low order bits of floating point numbers are effectively random. The presence of these random bits prevents lossless methods from achieving high compression rates. If some of the low order bits of floating point numbers can be removed (or approximated) without a significant impact on physics answers, we can achieve greater compression at the expense of lost information. We believe a compression method suitable for in-memory compression should be able to run in both lossless and lossy modes, and to be amenable to implementation in hardware with minimal resources and power consumption.

We classify lossy compression schemes into three broad categories: general lossless schemes like gzip and bzip2, transform coding approaches such as those based on wavelet or 
discrete cosine bases, and predictive coding schemes like the two methods we apply in this paper. We do not consider general lossless compression methods in this paper as they are limited in the compression rates that can be achieved and tend to be less effective for floating point data. Although transform coding is traditionally used for lossy compression, these methods have two main drawbacks: First, bit-for-bit lossless compression with these schemes can be difficult to achieve due to the subtleties of floating-point arithmetic, including rounding modes and error, catastrophic cancellation, order-of-evaluation dependence, extended precision, etc. Second, hardware implementations for the in-memory use-case are likely to use too many resources to be practical.

For these reasons, and for speed, we focus on predictive coding approaches, in which each floating-point number is predicted based on a trend of recently encoded values. The prediction residual (difference between actual and predicted value) tends to be small and can generally be encoded using fewer bits than the original floating-point value. The number of recent values is generaly quite small for a $1 \mathrm{D}$ predictor and therefore a hardware implementation can be quite compact. Typically, a lossless compression scheme is then used to compress the residuals so that the overall approach is bit-for-bit lossless. Lossy compression can be achieved by ignoring low order bits of the input values, resulting in smaller residuals and higher compression rates. For example, applying lossless compression after zeroing the bottom 32 bits of a double precision number usually produces total compression ratios around 3-4X. Finally, the errors introduced by predictive schemes are independent of neighboring values, usually being the result of truncation or rounding which occur already in fixed precision floating point computations, whereas the errors introduced in transform coding (e.g., Gibbs ringing) have spatial extent and exhibit spatial correlations.

In this paper we study two compression algorithms designed for floating-point data: Samplify's APAX (APplication AXceleration) encoder [16,17] and the fpzip $[7,18]$ compressor developed at LLNL. The APAX algorithm has previously been applied to climate data [19], computed tomography x-ray samples [20], and a variety of integer and floating-point datasets [21]. We describe these two compressors in parallel to highlight their similarities and differences.

\subsection{The fpzip and APAX Compressors}

When used for lossy compression, APAX and fpzip both begin by quantizing the value $f$ to be encoded, in effect reducing its precision. Quantization in $A P A X$ involves converting a block of $N$ consecutive values (usually $N=256$ ) to a signed integer representation, which can be thought of as aligning the floating-point values in a block to a common largest exponent. If exponent differences are large, this uniform quantization step may result in some loss of precision for the smallest (in magnitude) values in the block. After exponent alignment, each signed value is treated as a 32-bit integer $\hat{f}$. For (64-bit) double-precision data, this implies that the bottom 32 bits of the significand are discarded. This design decision was motivated by performance reasons - by modifying $A P A X$ to use 64-bit integer arithmetic, such truncation could be avoided, possibly at the expense of slower compression. APAX then quantizes the integer $\hat{f}$ uniformly to $\bar{f}=\operatorname{round}\left(\frac{\hat{f}}{q}\right)$, where $q$ is a quantization level either specified by the user or computed adaptively by $A P A X$ to meet a target coding rate.

fpzip delays the integer conversion and leaves the values in their floating-point representation, quantizing the significand instead. fpzip restricts $q$ to be an integer power of two, which effectively leads to truncation of the significand by discarding (zeroing) some fixed number of least significant bits. Setting $q=1$ guarantees entirely lossless compression. This non-uniform quantization allows the relative error to be bounded in fpzip, whereas within each APAX block the absolute error is bounded. Quantization is the only potential source of loss in both compressors.

Following quantization, each value is predicted as a linear combination of recently encoded values. Both compressors rely on polynomial interpolation for prediction, with fixed integer polynomial coefficients. APAX uses univariate Lagrange polynomials of degree 0 and 1 , allowing linear polynomials (or any function with $\frac{\partial^{2} \bar{f}}{\partial x^{2}}=0$ ) to be reproduced. That is, the unknown value $\bar{f}[x]$ is predicted in terms of the $n$ previous, known values by solving $\sum_{i=0}^{n}(-1)^{i}\left(\begin{array}{c}n \\ i\end{array}\right) \bar{f}[x-i]=0$. The "best" polynomial degree $n-1$ is chosen locally by monitoring its effect on compression.

fpzip exploits correlations in more than one dimension using the Lorenzo predictor [22], which over a 3D domain reproduces trivariate quadratic polynomials (or any function for which $\left.\frac{\partial^{3} f}{\partial x \partial y \partial z}=0\right)$. In $3 \mathrm{D}$ this predictor solves $\sum_{i, j, k \in\{0,1\}}(-1)^{i+j+k} f[x-i, y-j, z-k]=0$ for $f[x, y, z]$, and thus requires buffering a whole $2 \mathrm{D}$ "slice" from the $3 \mathrm{D}$ domain. Note that this predictor uses only additions and subtractions of known values.

Given the true floating-point value $f$ and its prediction $p$, fpzip converts $f$ and $p$ to integers $\bar{f}$ and $\bar{p}$ via a monotonic mapping that treats the binary floating-point number as a sign-magnitude integer. This step is not needed in $A P A X$, where the integer conversion occurs earlier. Both compressors then compute an integer residual $\bar{r}=\bar{f}-\bar{p}=s\left(2^{e}+d\right)$ with sign $s \in\{-1,0,+1\}$, exponent $e$, and $e$-bit difference $d \in\left\{0, \ldots, 2^{e}-1\right\}$. The bits of $d$ generally exhibit no correlation and are transmitted verbatim. However, the sequence of "signed exponents" $\tilde{e}=s(e+1)$ tends to be highly correlated (if not necessarily peaked around zero). APAX exploits spatial correlations by encoding differences between consecutive exponents in small groups. fpzip, on the other hand, models the non-uniform distribution of exponents and encodes each $\tilde{e}$ independently using a fast entropy coder.

\subsection{APAX Profiler}

A compression algorithm that takes advantage of "signal" characteristics may provide better compression ratios than one that does not. The spatial wavelength dependence of data arrays may be examined with popular applications like MatLab and Mathematica. APAX includes a profiler that allows a user to investigate the compressibility of their data and APAX's tradeoffs between bit rate and signal quality. Figure 1(left) illustrates the APAX rate-distortion curve of the pF3D ion acoustic wave array. The profiler suggests a Recommended Operating Point (ROP) where the Pearson's correlation coefficient between the original data $x$ and the APAX-decoded data $\tilde{x}$ is 0.99999 ("five nines"). In Figure 1(right), the profiler compares the input signal spectrum (upper curve) to the residual spectrum (lower curve) and quantifies the spectral margin at the ROP. 

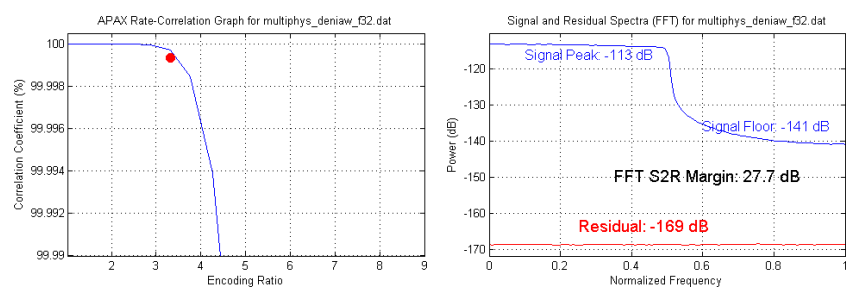

Figure 1: APAX profiler results for a pF3D array representing ion acoustic waves. The profiler shows (left) the recommended compression level and (right) the corresponding power spectra.
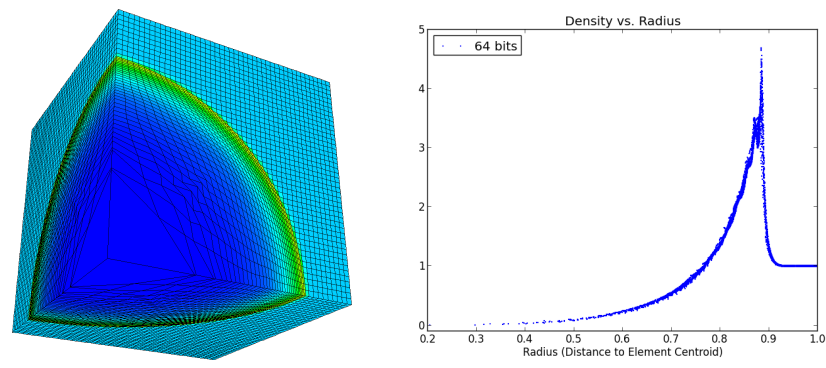

Figure 2: An overview of the LULESH shockhydrodynamics simulation. (left) Density field showing the shock wave and mesh deformation. (right) Scatter plot of density vs. radial displacement from the origin for every element in the mesh.

\section{SIMULATION \& VALIDATION METHODS}

\subsection{LULESH}

LULESH is a shock hydrodynamics mini-application developed for use in evaluation of current and future computer systems and proposed programming models [23-25]. LULESH solves the Sedov blast wave test problem - a point explosion surrounded by an initially uniform surrounding gas. The gas consists of one material and is modeled in three dimensions using a Lagrangian (moving mesh) formulation. Figure 2 depicts two key features of the simulation - the deformation of mesh elements and the shock wave.

LULESH solves the inviscid compressible Navier-Stokes equations in the Lagrangian formulation. A staggered mesh approximation [26] with single point quadrature for elementcentered thermodynamic quantities such as density and pressure is utilized. Kinematic variables such as position and velocity are defined at mesh nodes. The Sedov problem presents an interesting use case for compression, as most field values vary slowly over most of the domain, but change quickly and abruptly near the shock.

For the Sedov problem, the two key physics requirements are that the blast wave should be spherical, and the shock radius versus time should match the analytic solution. In this paper we evaluate the symmetry of the shock by comparing the field values as a function of radius for various compression levels and methods, to the results obtained with a double precision simulation with no compression. We assess physical accuracy by estimating shock position as the distance to the centroid of the element with maximal density,

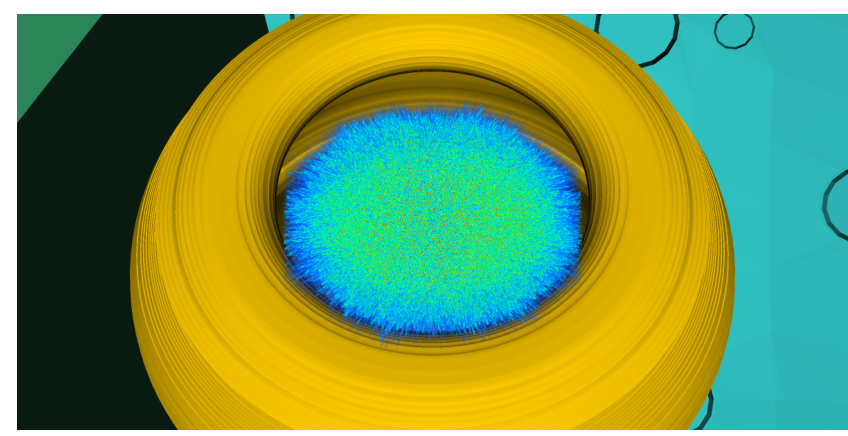

Figure 3: The figure shows the intensity of the laser beam as it enters the hohlraum. The hohlraum is a can-like object with the fusion target at its center. The intensity increases as the color changes from blue to green to yellow to red. The bright spots embedded in a lower intensity background are a design feature of NIF beams.

and comparing that value between runs with and without compression. Although this measure is not the most sophisticated way to assess shock position, it is simple and can be consistently applied across LULESH runs at varying mesh resolutions. Finally, we measured differences in internal energy between compression and non-compression runs to validate that compression wasn't violating the expected behavior of the LULESH simulation as represented by full double precision runs at different mesh resolutions.

\section{2 pF3D}

The National Ignition Facility (hereafter NIF; $[27,28]$ ) is an NNSA experimental facility that houses the world's most powerful laser. One of the key goals of the NIF is to compress a target filled with deuterium and tritium to a temperature and density high enough that fusion ignition occurs.

The intensity of NIF beams exceeds $10^{15} \mathrm{~W} / \mathrm{cm}^{2}$ in the brightest spots. When intensities are this high, it is possible for the laser to couple to fluctuations in the plasma density and backscatter a significant fraction of the laser light. pF3D [29-31] is a multi-physics code that simulates interactions between laser beams and the plasma in NIF experiments. $\mathrm{pF} 3 \mathrm{D}$ is used to evaluate proposed target designs and to pick the ones with acceptably low levels of backscatter.

pF3D zones are roughly the size of the laser wavelength $(0.35 \mu \mathrm{m})$ while the plasmas of interest are several mm across. Simulations of the full path of a single laser beam require 50 billion or more zones. Simulations of five interacting beams may require more than a trillion zones. $\mathrm{pF} 3 \mathrm{D}$ has been used to run simulations with $64 \mathrm{k}$ or more cores on IBM Blue Gene/L, IBM Blue Gene/P, and Cray XE-6 systems. Work is underway to run a million core simulation on an IBM Blue Gene/Q system.

Figure 3 shows the intensity pattern of the laser beam as it enters the target in a NIF experiment. The bright spots are referred to as speckles. Speckles are narrow transverse to the laser propagation direction and extend for many laser wavelengths in the propagation direction. The laser is designed so that the speckles in the beam move around in time. The plasma temperature and density respond to the intensity averaged over time, so they see a smooth beam.

Backscattered light can be generated in the brightest speck- 

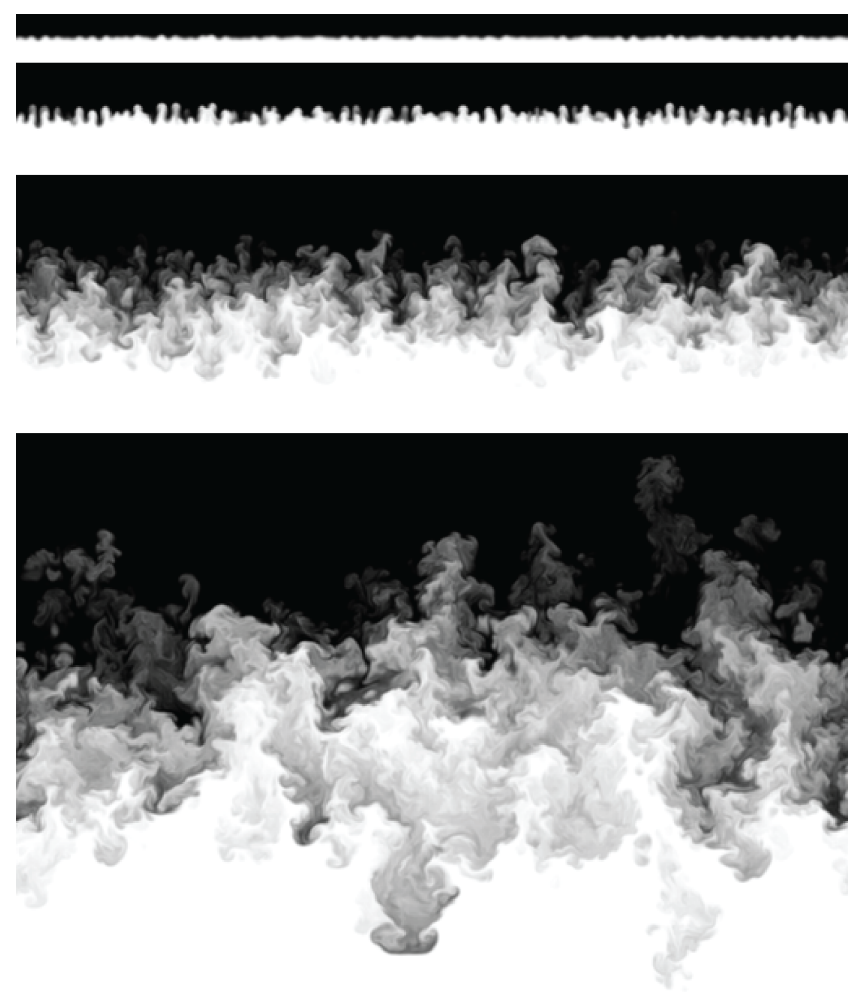

Figure 4: Density fields are shown from a Miranda simulation of the Rayleigh-Taylor instability at (from top to bottom) $t / \tau=1,2.5,10,20$.

les and grow in strength faster than the speckles move. The laser light will refract when there is a density gradient. Speckles are long enough that a small change in density can shift a speckle sideways by a significant fraction of its width.

The locations of the speckles are extremely sensitive. For example, the fused multiply-add instruction of the PowerPC processor (with internal registers that are longer than 64 bits) produces results sufficiently different from x86_64 processors to cause speckles to move sideways by a zone or more. Compiler optimizations that change the order in which summations are carried out also cause speckles to move.

As a result of this high sensitivity to such small variations, validation of new versions of $\mathrm{pF} 3 \mathrm{D}$ relies on comparisons of the total transmitted and reflected light as a function of time (the total light is insensitive to the exact placement of speckles) and on intensity histograms. This paper uses the same physics-based validation methods to assess the impact of lossy compression on pF3D.

\subsection{Miranda}

Miranda [32,33] is a Navier-Stokes code used to simulate a range of hydrodynamic problems with higher-order accuracy. It uses spectral methods or compact differencing to resolve turbulent structures with minimal dissipation. Dissipation is added at high wavenumbers through the use of artificial fluid properties [34], which act as a large-eddy simulation (LES) subgrid model. Miranda has run simulations using over $64 \mathrm{k}$ cores on Blue Gene/L systems.

The Miranda test problem simulates the growth of the
Rayleigh-Taylor instability (RTI). Simulations of the RTI typically start with small-scale perturbations on the interface separating two fluids of different densities. The high density fluid is on top of the low density fluid - an unstable situation. The perturbation amplitudes grow, neighboring perturbations merge, and, eventually, turbulent mixing occurs. This inverse cascade of scales, from the initial short wavelength perturbation to large wavelengths at late times, requires high-order accuracy to ensure relevant features are not removed through dissipative numerics or influenced by the amplification of numerical noise.

The test problem is physically unstable to all perturbation wavelengths. Filtering is employed to damp the growth of modes with short wavelengths. The damping is quite strong at a wavelength of 2 zones, but drops quickly as the wavelength increases. An important point to remember when investigating compression schemes is that the physics will amplify numerical perturbations introduced by compression as well as deliberately imposed perturbations. This means that the wavelength spectrum and step-to-step coherence of the perturbations produced by compression matter, in addition to the compression ratio.

The incompressible simulations shown here are initialized with narrowband Gaussian perturbation spectra peaked at 8 grid cells per wavelength $\left(\lambda_{0}\right)$ and an RMS amplitude of 0.1 grid cells. This allows the instability to begin in its linear stage. The two fluids have densities of $\rho_{1}=1$ and $\rho_{2}=3$, and gravitational acceleration of $g=1$, providing a time scaling of $\tau=\sqrt{\lambda / A g}$, where $A=\left(\rho_{2}-\rho_{1}\right) /\left(\rho_{2}+\right.$ $\left.\rho_{1}\right)=0.5$ is the Atwood number. Density fields from a $512^{2} \times 1024$ simulation using 1024 processors are shown in Figure 4 . In the upper two images, at $t / \tau=1$ and 2.5 , the perturbations - just barely noticeable in the first image are growing independently at an exponential rate. By $t / \tau=$ 10, shown in the third image, perturbations have merged, producing larger scales and mixed fluid. At $t / \tau=20$, shown in the lower image of Figure 4, the layer has entered into an apparent turbulent state.

An important quantity in assessing RTI simulations is the mixing layer thickness, $h$, which is expected to behave as $\dot{h}^{2}=4 \alpha A g h$ when the layer reaches a self-similar state at late times. Another important characteristic at late times is the spectrum of perturbations as a function of spatial frequency. These physically motivated quantities will be used to assess the differences between compressed and uncompressed simulations.

\section{RESULTS}

This section presents results on the acceptable level of compression in the LULESH, Miranda, and pF3D test problems. Simulations with and without compression are compared using the physics metrics mentioned in the earlier discussion. Simulations may be sensitive to the details of how compression is performed. As a result, the acceptable level of compression for a given test problem may differ between different compression algorithms.

The codes have been modified so that they compress and decompress variables at the end of each time step, with each variable represented on a (logical) Cartesian 3D grid. This procedure approximates "memory compression," which, if actually in place between the memory and the cache, would occur several times per time step. We also ran a Miranda test where the compression function was called at the lower 
frequency of checkpoint dumps to approximate "disk compression".

We report the minimum, maximum, and average compression ratios over all domains. The minimum compression ratio is the most important measure when using "memory compression." The process with the lowest compression ratio will have the hardest time fitting in the available memory, and it will spend the most time reading and writing memory. All three of these codes use a bulk synchronous programming model, so the slowest process controls the performance. Efficient parallel I/O packages have performance that is dependent on the total number of bytes written, not on the number contributed by each process. With an I/O package of this sort, the average compression ratio is the important quantity.

\subsection{LULESH}

In this section we explore the effects of compression on the accuracy of the LULESH Sedov blast wave simulation. We performed two studies: in the first study we varied the mesh size and ran fpzip over a full range of precisions, to $t=0.002$. The energy at $t=0$ was scaled such that the shock wave traversed nearly to the boundary. In the second study, we ran with both $A P A X$ and fpzip on a $105^{3}$ mesh to time $t=0.05$ at a small set of target compression rates $(A P A X)$ or precisions (fpzip). We scaled energy at $t=0$ such that the shock propagated to the boundary, in order to assess if errors increased over time. We assessed shock position in a manner similar to Tasker et al. [35]. Figure 5 (left) shows density vs. radius for all elements in a $105^{3}$ simulation, with the shock position taken as the radius at the maximum density value in the plot.

In the first study, we ran a series of simulations with fpzip at precisions from 24 to 64 bits in steps of 4 bits on several mesh sizes. Figure 5(middle) shows that mesh size has a larger effect on shock position than compression rate. However, Figure 5(right) shows that the root mean square (hereafter RMS) error is actually slightly larger for higher resolution meshes. We note that higher resolutions require smaller time steps and that may cause higher RMS errors due to a larger number of applications of compression. For example, the $45^{3}$ problem completes in 1496 time steps, but the $120^{3}$ problem requires 4272 time steps. Our assessment is that with respect to shock position and RMS error in individual density values, the LULESH Sedov simulation has correct behavior down to 48 bits, and that in several aspects precisions as low as 32 bits are sufficient. We confirmed this assessment by measuring internal energy loss in longer-running simulations as we present below.

In the second study we ran simulations at $105^{3}$ resolution to $t=0.05$. This study showed that the shock position was accurately captured at fpzip precisions down to 32 bits, and $A P A X$ rates up to $4 \mathrm{X}$. The density vs. radius plot in Figure 5 (left) shows density vs. radius for fpzip precisions of 24 and 64 bits (lossless), showing a failure mode when the precision is too low. The shock radius is smaller than in the higher precision runs, indicating that energy has been lost and the shock is trailing the converged full-precision solution. Figure 6 shows zoomed in views of the density field at the two precisions showing that the shock is no longer spherical. We note that fpzip may introduce a bias as it first discards significand bits, effectively rounding towards zero. However, we also found that at high compression rates $A P A X$ also
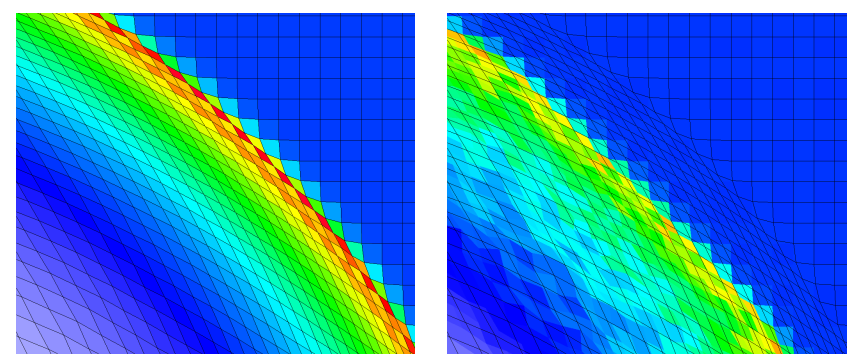

Figure 6: (left) A zoom in of the density field at the shock in a $105^{3}$ mesh at full precision. (right) The shock at 24 bits of precision, showing that a significant amount of noise is introduced, although this noise is symmetric. In both images, blue indicates low density, and red indicates the highest density.

\begin{tabular}{|c|c|c|c|}
\hline compressor & ratio & energy & result \\
\hline fpzip 64-bit & $2.0 / 2.5 /$ & 0.0 & pass \\
\hline fpzip 48-bit & $2.8 / 3.7 / 9.7$ & $-1 \times 10^{-6} \%$ & pass \\
\hline fpzip 32-bit & $3.8 / 6.5 / 14.7$ & $-0.07 \%$ & pass \\
\hline fpzip 24-bit & $\mathrm{N} / \mathrm{A}$ & $\mathrm{N} / \mathrm{A}$ & fail \\
\hline$A P A X 2 \mathrm{X}$ & $2.4 / 3.1 / 19.2$ & $+6 \times 10^{-6} \%$ & pass \\
\hline$A P A X 3 \mathrm{X}$ & $3.0 / 3.5 / 19.2$ & $-0.002 \%$ & pass \\
\hline$A P A X 4 \mathrm{X}$ & $4.0 / 4.5 / 19.0$ & $-0.01 \%$ & see text \\
\hline
\end{tabular}

Table 1: This table shows the compression ratios achieved for the LULESH runs for mesh size $105^{3}$. The 'ratio' column shows the minimum, mean, and maximum compression ratios for all fields in the problem. The value for mean compression is the ratio between the storage required for all fields, divided by the total compressed size of all fields. The 'energy' column records the percent of internal energy lost (or gained), with respect to the full precision run. The compression algorithm and setting for each run is shown and is labeled by whether it passes the physics criteria.

loses energy, presumably due to the scaling process it uses to convert floating point values into 32 -bit integers before compressing them.

Table 1 shows the compression ratios and final internal energy change achieved for LULESH runs using both fpzip and $A P A X$ compression for the second study. We see that over a fairly large range of compression rates and precisions that minimal energy is lost. The $4 \mathrm{X} A P A X$ run showed good agreement with internal energy and shock position but seeded non-physical noise in the nodal positions ahead of the shock, so we did not deem that a total 'pass', nor a total 'fail'. The Sedov problem is stable, and it appears that this noise in nodal positions in front of the shock does not impact the shock position adversely, at least up to the time we ended the simulation.

A limitation of our results is that the Sedov problem is a simple test case. The requirements of simulations involving more complicated geometries and multiple materials may have more restrictive requirements with respect to lossy compression. In addition, LULESH is a mini-app and contains fields whose sole purpose is to reproduce data movement patterns in larger codes. Since these fields are unitvalued and do not impact simulation results we do not in- 

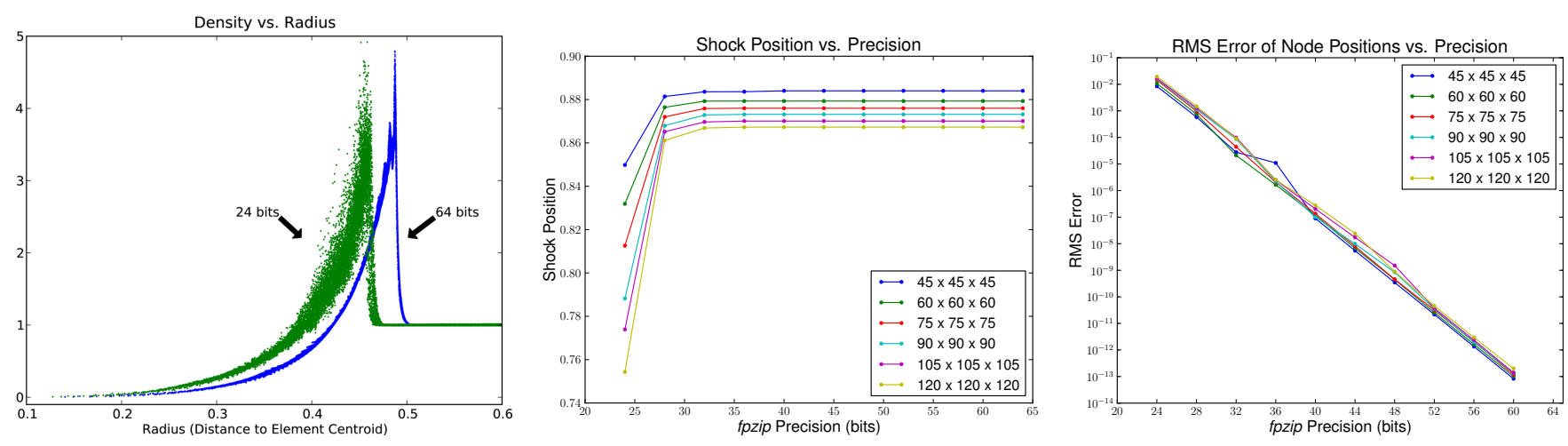

Figure 5: (left) Density plotted against radius at $t=0.02$, for $f p z i p$ precision values of 24 and 64 bits, and a $105^{3}$ mesh size. When retaining only 24 bits of precision, a significant amount of energy is lost, causing the shock to be delayed. (middle) Shock position as a function of mesh size and fpzip precision. As we increase mesh precision, the apparent change of shock position is larger than that induced by most precision settings. (right) Root mean square error of nodal positions with respect to a run with no compression.

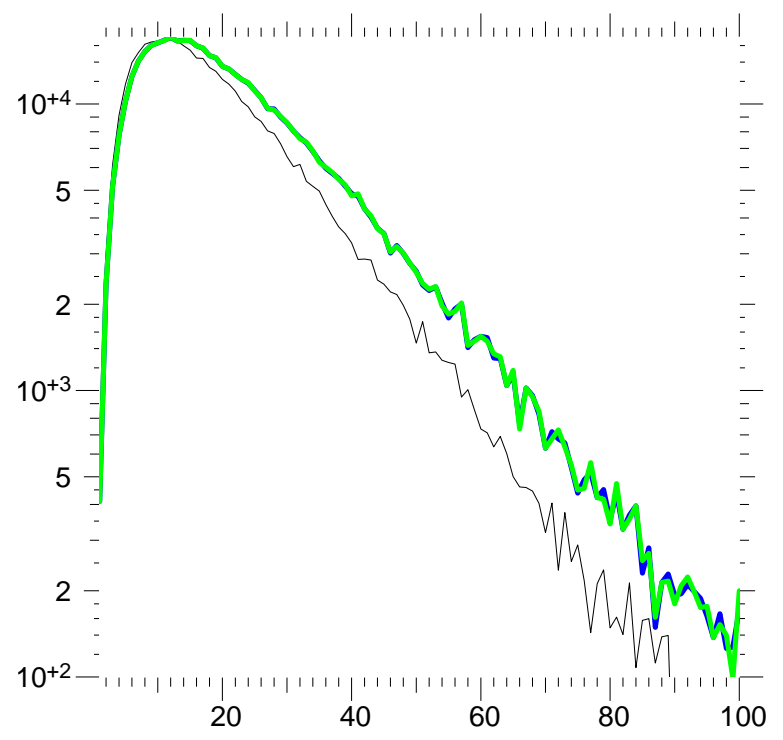

Figure 7: The figure shows a histogram of the amount of laser energy per bin as a function of the laser intensity. The beam has crossed the full extent of the plasma. The black curve is from a run using 24-bit fpzip, the blue curve is from a run using 32bit fpzip, and the green curve is from a a run using uncompressed double precision variables. The blue and green curves overlay one another. The curves for runs using APAX compression are very similar and are not shown to avoid visual clutter.

clude them in our compression ratio data. Finally, LULESH does not converge to a shock position cleanly, and up to mesh resolutions of $175^{3}$ we saw the shock position continuing to vary slightly.

\section{2 pF3D}

The $\mathrm{pF} 3 \mathrm{D}$ test problem simulates the propagation of a laser beam through a plasma. The plasma is divided into $1 \times 2 \times 36$ equal domains - one for each of the $72 \mathrm{MPI}$ processes. There are a total of $256 \times 1024 \times 756$ zones, each of which is $2 \times 2 \times 2$ wavelengths in size. Some of the laser light is absorbed, some is backscattered by Stimulated Brillouin Scattering (SBS), and some reaches the exit plane. When compression levels are high, some of the laser energy is "eaten by numerical gremlins." The total amount of laser energy reaching the exit plane and the total amount of SBS crossing the entrance plane are key integral measures of what happens in a simulation. A more detailed metric is the amount of laser energy as a function of laser intensity.

Figure 7 shows a histogram of the amount of laser energy per bin as a function of the laser intensity. The histogram is made after the laser has crossed the full extent of the plasma, so energy has been lost due to absorption, backscattered light, and numerical effects. All of the pF3D simulations used double precision arithmetic. The blue (32-bit fpzip) and green (lossless $f p z i p$ ) curves are effectively identical. The black curve (24-bit fpzip) is significantly lower at all but the lowest intensities and indicates that compression has led to the loss of laser energy. Compression is applied to the electric field of the laser, but the laser energy depends on the square of the field. A compression scheme that preservers the integral of the electric field can still significantly alter the laser energy. The compression scheme may also alter the direction in which portions of the laser beam travel. Laser energy propagating at an angle to the $z$-axis will travel a greater distance and suffer more absorption before reaching the exit plane.

Figure 8 shows a histogram of the amount of backscattered energy per bin as a function of the intensity at the entrance plane. The blue (32-bit fpzip) and green (uncompressed) curves are effectively identical. The black curve (24-bit fpzip) has significantly lower energy at all intensities, a natural consequence of the reduced laser energy reaching the back of the simulation.

Table 2 shows the compression ratios achieved for the pF3D runs. The 64-bit fpzip run used lossless compression and only achieved a $23 \%$ reduction in data size. The 48 -bit and 32-bit fpzip runs passed the physics criteria while the 24-bit fpzip run failed due to too much loss of energy in the transmitted laser light. The simulations using APAX compression passed the physics criteria for $2 \mathrm{X}, 3 \mathrm{X}, 4 \mathrm{X}, 5 \mathrm{X}$, and up to $6 \mathrm{X}$ but failed at $7 \mathrm{X}$ due to too much increase in the 


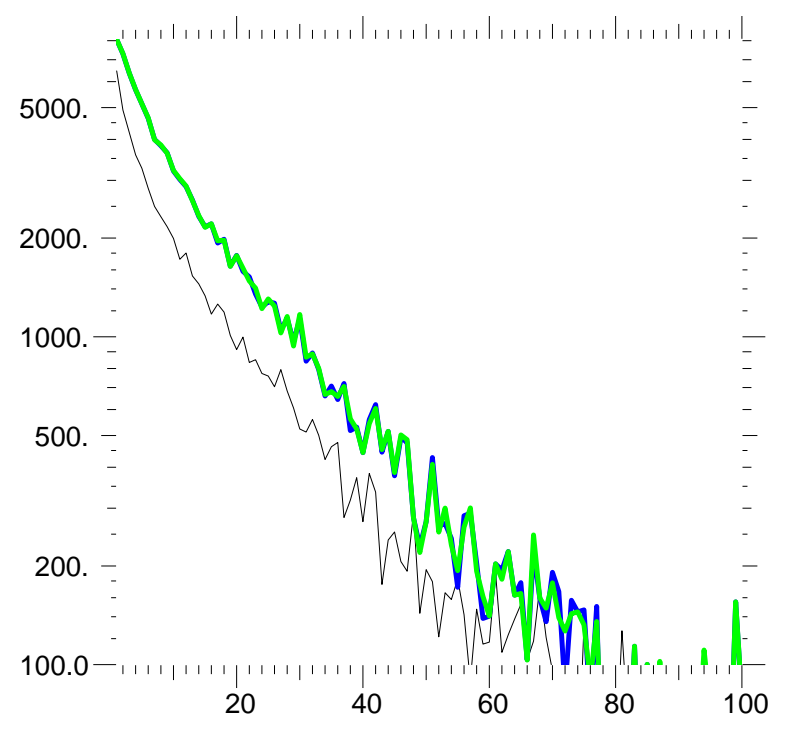

Figure 8: The figure shows a histogram of the amount of backscattered energy per bin as a function of the laser intensity. The histogram is made at the entrance plane where the backscattered light is at its maximum. The black curve is from a 24-bit fpzip run, the blue curve is from a 32-bit fpzip run, and the green curve is from a run without compression. The blue and green curves overlay one another.

\begin{tabular}{ccrc}
\hline compressor & ratio & energy & result \\
\hline fpzip 64-bit & $1.27 / 1.29 / 1.32$ & 0 & pass \\
fpzip 48-bit & $1.86 / 1.91 / 1.97$ & 0 & pass \\
fpzip 32-bit & $3.46 / 3.66 / 3.89$ & $-0.2 \%$ & pass \\
fpzip 24-bit & $5.89 / 6.57 / 7.37$ & $-37 \%$ & fail \\
\hline$A P A X 2 \mathrm{X}$ & $2.19 / 2.22 / 2.26$ & 0 & pass \\
APAX 3X & $3.03 / 3.03 / 3.09$ & 0 & pass \\
$A P A X$ 4X & $4.06 / 4.06 / 4.14$ & $0.1 \%$ & pass \\
$A P A X$ 5X & $5.06 / 5.07 / 5.16$ & $0.9 \%$ & pass \\
$A P A X 6 \mathrm{X}$ & $6.09 / 6.09 / 6.20$ & $1.2 \%$ & pass \\
$A P A X 7 \mathrm{X}$ & $7.10 / 7.11 / 7.22$ & $10.7 \%$ & fail
\end{tabular}

Table 2: This table shows the compression ratios achieved for the pF3D runs. The 'ratio' column contains minimum, mean, and maximum compression rates. The ratio is the number of bytes in the uncompressed arrays divided by the number of bytes in the compressed arrays. Each run is characterized as passing or failing the physics criteria, or crashing. The 'energy' column shows the percent difference in the backscattered energy relative to the uncompressed run.

transmitted laser light. We also ran pF3D with float (32-bit) precision and no compression. This run passed the physics criteria and could be thought of as achieving $2 \mathrm{X}$ compression. The run with 48-bit fpzip also achieved roughly $2 \mathrm{X}$ compression, but had higher precision than the run using float precision.

\subsection{Miranda}

To compare the effects of lossy compression on the development of the RTI, simulations with various levels of com-

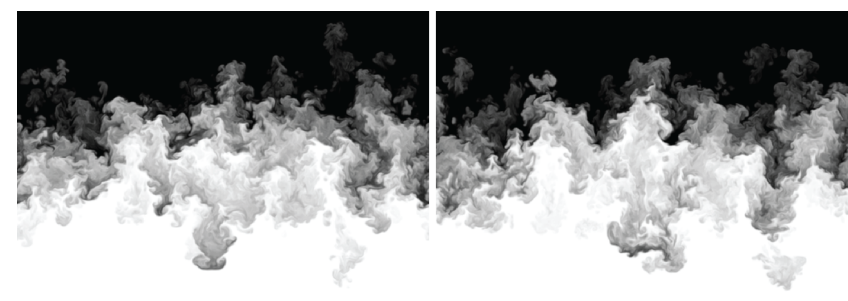

Figure 9: Density field at the end of the simulation from the reference (64-bit) run (left) and the $A P A X$ $5 X$ case (right), which have nearly the same mixinglayer thickness and turbulence characteristics.

pression were run on $512^{2} \times 1024$ grids using 1024 processors. After each time step, the density and the three components of velocity are compressed and decompressed using fpzip or $A P A X$. This results in $\sim 10,000$ lossy compression steps over the course of the simulation.

In the case of fpzip, the compression step truncates the 64-bit data to either 48,40 , or 32 bits. Larger compression rates are achieved $(\sim 4 \mathrm{X})$ in regions where the flow field is relatively uniform (lossless compression is applied after the numbers are truncated). To test the effect of compressed checkpoint files, an additional test was run calling the 32bit compression step every $500^{\text {th }}$ time step, resulting in 16 compress/decompress calls over the course of the simulation. In contrast, $A P A X$ was used in three simulations with $2 \mathrm{X}$, $4 \mathrm{X}$, and $5 \mathrm{X}$ compression at each time step.

Images of the mixing layer at the end of the simulation $(t / \tau=22)$ are shown in Figure 9 from the 64-bit case (left) and the APAX $5 \mathrm{X}$ case (right). While there are differences between the two images, the quantitative metrics presented below show that the integral quantities and the turbulence state are nearly identical.

The thickness of the mixing layer, $h$, is plotted in Figure 10 as the lower group of lines. The reference simulation (64-bit) is plotted in black while the 40- and 32-bit fpzip compression cases (called each time step) are plotted in blue and red, respectively. The $A P A X 5 \mathrm{X}$ case is plotted as a green dashed line. The other four cases considered are all plotted in gray, since their differences with each other and with the 64-bit curve are minor. The simulations were stopped once the dominant wavelength approached the size of the simulation domain. The 32-bit fpzip case is the only one that failed outright, as it crashed at $t / \tau \approx 14$ when it became numerically unstable. The other cases, and even the 32-bit fpzip case before crashing, differed little from the reference simulation. At the time the 32-bit fpzip case crashed, its mixing-layer thickness was $2.4 \%$ larger than the reference simulation. The 32-bit fpzip case where compression was only applied every $500^{\text {th }}$ time step ran without issue and differed by $0.38 \%$ in mixing-layer thickness at the end of the simulation $(t / \tau=22)$. The growth rates are also shown in Figure 10 as the upper set of lines with the same color scheme. At early times, when the layer is growing exponentially, there are no differences between the seven curves. Once nonlinear growth begins, after $t / \tau>3$, small differences are noticeable. These differences are minor and all cases show the expected $\dot{h} \propto t$ self-similar growth beyond $t / \tau>6$. Near the end of the simulation the fpzip 40-bit case and the APAX 5X case had 5-7\% smaller growth rates, re- 


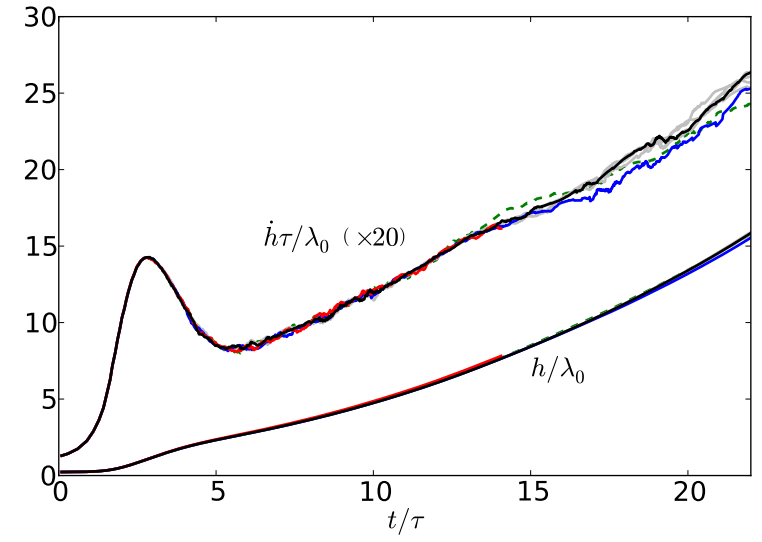

Figure 10: Mixing layer thickness (lower curves) and growth rate of the mixing layer (upper curves) from a reference (64-bit) calculation (black), an fpzip 32-bit compression case (red), an fpzip 40-bit compression case (blue), and an $A P A X$ $5 \mathrm{X}$ case (green dashed). The grey curves include a 48-bit fpzip case, a 32-bit fpzip case where compression is applied every $500^{\text {th }}$ step, and $A P A X$ with $2 X$ and $4 X$ compression at every time step. Small differences are observable in the 32- and 40-bit fpzip cases. The 32-bit fpzip case crashed at $t / \tau=14$.

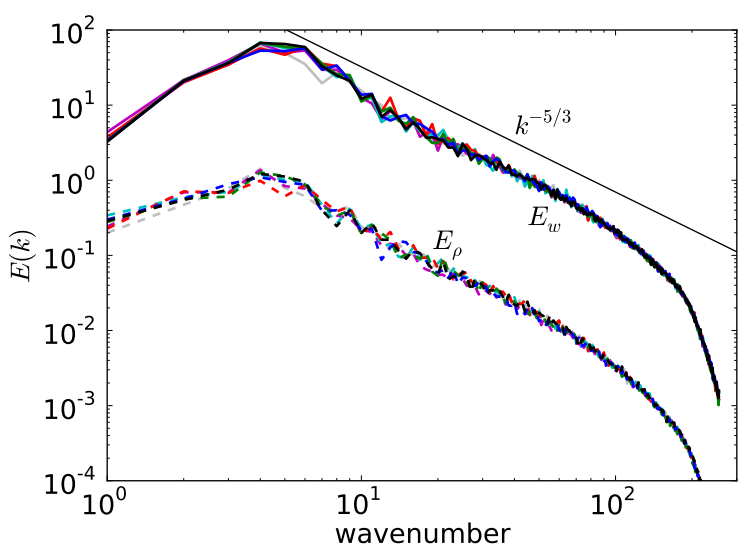

Figure 11: Energy spectrum of vertical velocity (solid) and density (dashed) at the mid-plane of the mixing layer at $t / \tau=22$. All cases are shown except the 32-bit fpzip case, which did not reach this time. The spectra are very similar and feature a $k^{-5 / 3}$ inertial range.

sulting in $1.9 \%$ and $0.49 \%$ smaller mixing-layer thickness, respectively. Since these growth rates are systematically smaller, rather than simply having different random fluctuations like the other cases (gray curves), it is likely that the mixing-layer thickness difference will compound and become unacceptable if the simulation were run later in time (requiring a larger initial domain). The differences in mixing-layer thickness at the end of the simulation are listed in Table 3.

The two-dimensional energy spectrum from the plane centered within the mixing layer is shown in Figure 11 at $t / \tau=$ 22 for both the vertical velocity (solid) and the density

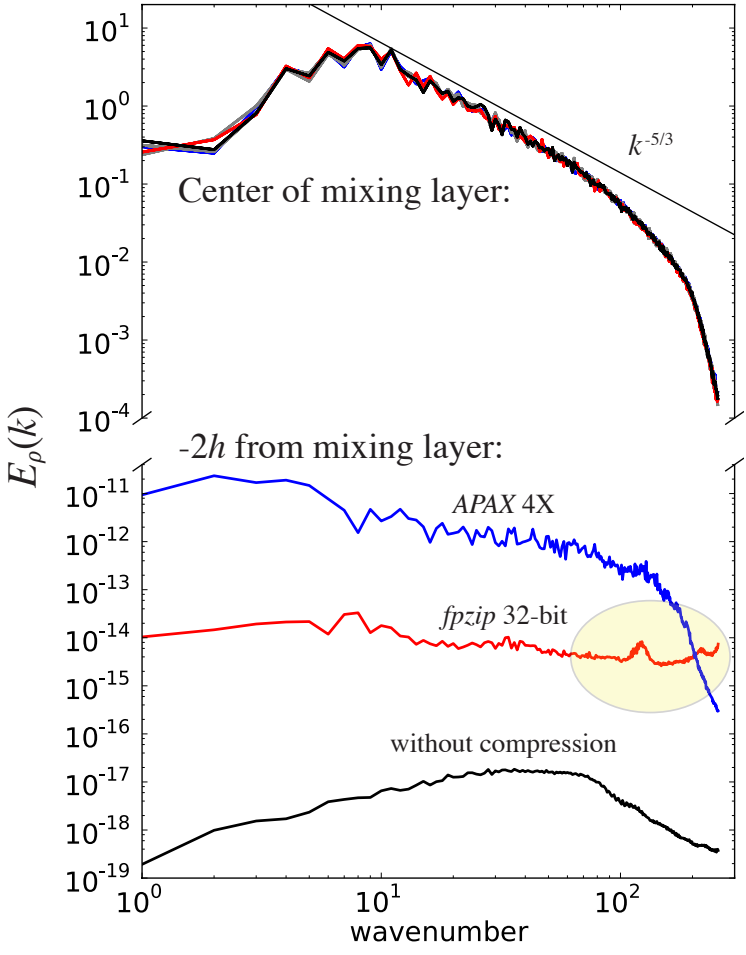

Figure 12: Energy spectrum of density at the midplane of the mixing layer (top set of curves) and at a distance $-2 h$ from the mixing layer (bottom set of curves) at $t / \tau=14$. The 32-bit fpzip case exhibits high wavenumber features that cause the problem to become numerically unstable.

\begin{tabular}{cccrc}
\hline compressor & ratio & loss & thick. & result \\
\hline fpzip 48-bit & $1.8 / 3.3 / 4.6$ & $5.9 \mathrm{E}-12$ & $0.07 \%$ & pass \\
fpzip 40-bit & $2.3 / 4.3 / 6.3$ & $1.7 \mathrm{E}-09$ & $1.9 \%$ & pass \\
fpzip 32-bit & $3.2 / 4.5 / 6.5$ & $4.1 \mathrm{E}-07$ & N/A & crash \\
\hline$A P A X 2 \mathrm{X}$ & $2.1 / 2.3 / 2.6$ & $3.3 \mathrm{E}-10$ & $0.007 \%$ & pass \\
$A P A X$ 4X & $4.0 / 4.1 / 4.3$ & $3.6 \mathrm{E}-06$ & $0.19 \%$ & pass \\
$A P A X$ 5X & $5.1 / 5.3 / 5.4$ & $4.7 \mathrm{E}-05$ & $0.49 \%$ & pass
\end{tabular}

Table 3: This table shows the compression ratios achieved for selected Miranda runs. The 'ratio' column shows the minimum, mean, and maximum compression rate achieved throughout the simulation domain. The 'loss' column reports the RMS difference at each time step in the density field due to lossy compression divided by the mean density. The 'thick.' column shows the percent difference in mixing-layer thickness at the end of the simulation. Each run is characterized as passing the physics criteria, failing the criteria, or crashing.

(dashed) (the 32-bit case is not shown, as it did not reach this time). These spectra show the amount of energy contained in the various length scales within the problem. At large wavelength (low wavenumbers) the energy remains low enough that the physics are not influenced by the domain size. At the smallest scales (high wavenumbers) the energy is removed through viscous dissipation. In-between these scales is a power-law region, which, if the flow is turbulent, 


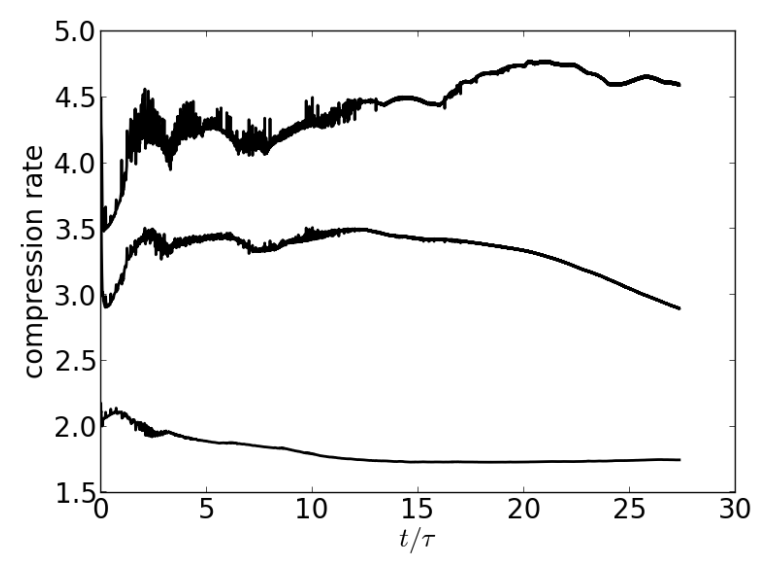

Figure 13: The compression ratio for an fpzip 48-bit compression run is shown. The three curves represent the maximum, minimum, and mean compression rate among the 1024 processes.

has a $-5 / 3$ slope. Aside from small fluctuations, the curves are nearly identical and all exhibit a $-5 / 3$ power-law for over a decade in wavenumbers, signifying turbulent behavior.

To further investigate why the fpzip 32-bit case crashed, Figure 12 shows density spectra at $t / \tau=14$. The top set of curves show the energy spectra of the density field at the center of the mixing layer from all of the cases considered. At this location the spectra are all nearly identical, showing the beginnings of a power-law range and a dissipation region at high wavenumbers caused by Miranda's LES filtering. The lower set of curves show the spectra from three simulations, taken from a plane located a distance $-2 h$ from the center of the mixing layer, where the flow field is relatively quiescent. The $A P A X 4 \mathrm{X}$ case produced a greater loss of precision than the fpzip 32-bit case, which is reflected in its larger energy level in this region. In the fpzip 32-bit case, however, a bump can be seen in the high wavenumber region that corresponds to approximately 5 grid cells per wavelength. This scale is large enough to remain after Miranda's filtering routines. This spectrum also shows an up-turn at the highest wavenumber portion of the spectrum. It is these features that accumulate and interact with Miranda's numerics that cause the problem to crash. This shows that precision is not the only factor to consider when choosing a compression scheme for "memory compression" one must also consider, or test, how compression will interact with the code's numerics.

The compression rate varied over the course of the simulation, within different regions of the simulation domain, and for the different fields being compressed. In the $A P A X$ $5 \mathrm{X}$ case, for example, the three velocity fields were all compressed to $5-5.5 \mathrm{X}$, while the density field started out at $14 \mathrm{X}$ compression in regions far from the mixing layer, and then reduced to $\sim 5 \mathrm{X}$ compression later in time. The rates were averaged across time and fields and are reported in Table 3. Also reported in this table is the loss that compression introduced at each time step. This is defined as the RMS difference in the density field after compression was applied and normalized by the mean density. This loss criterion was evaluated in the center of the mixing layer. As noted in the table, all of the runs passed the physics criteria except for

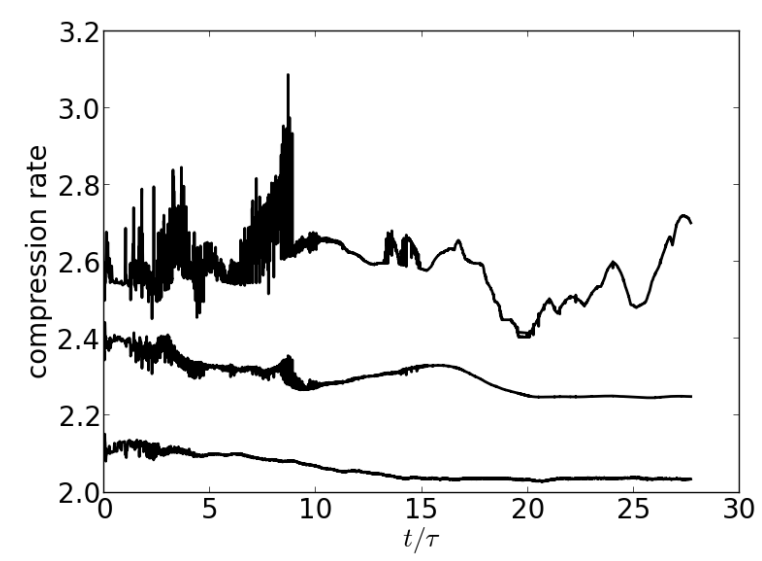

Figure 14: The compression ratio for a $2 \mathrm{X} A P A X$ compression run is shown. The three curves represent the maximum, minimum, and mean compression rate among the 1024 processes.

the 32-bit fpzip run.

The fpzip compression rate varied over the course of the simulation and at different regions within the simulation domain. Figure 13 shows the compression rate of all fields from a 1024 processor RTI simulation using Miranda and compressed with fpip in 48-bit mode on each time step. The compression rate begins at $\sim 2.2$ in processors near the mixing layer and at $\sim 4.5$ in processors farther away. Over time the minimum compression rate decreases, reaching a floor in some processes at 1.7. Over the course of the simulation, an average compression rate of 3.3 was achieved.

Figure 14 shows the compression rate of one of the velocity components from a 1024 processor RTI simulation using Miranda and APAX $2 \mathrm{X}$ compression on each time-step. The average compression rate is slightly above $2 \mathrm{X}$ due to the fact that the APAX coder converts all float values into a 32-bit signed integer representation before quantization. The minimum compression ratio never falls below 2.0 on any processor. A fixed rate compression scheme may be important for memory limited applications, in which a strict lower bound on compression rate is required.

\section{CONCLUSIONS}

This paper has examined the impact of lossy compression on three physics simulation codes - LULESH, Miranda, and pF3D. All three codes can be run with a lossy compression ratio of $3 \mathrm{X}$ or greater when using a frequency suitable for "memory compression." To our knowledge, this is the first study of the effects of applying lossy compression to the physics state of simulations as a strategy for mitigating the data movement bottleneck expected on future systems.

The LULESH study indicates that a broad range of compression parameters are valid for this run. The Sedov blast wave problem is a stable problem, and we expect that for unstable flows higher precision settings may be required. We also found that for LULESH, the mesh size changed the shock position more than the compression settings, until very low precisions broke down the spherical symmetry of the problem and seeded noise in the field values. Finally, we noted that $A P A X$ and fpzip were similar in their performance, indicating that compression schemes with either 
fixed rate or fixed precision modes are viable. We found that with $A P A X$, the per-block scaling of values did lead to unphysical noise in the nodal positions in front of the shock wave at a requested compression rate of $4 \mathrm{X}$. In future work, it would be interesting to thread the compression calls through the LULESH solver at the point when each field value is updated, as this would be closer to how memory compression would occur in practice.

The Miranda run with 32-bit fpzip failed when compressed at every time step but passed when run at the much lower frequency required for "disk compression." This run had a compression rate of $4.5 \mathrm{X}$, only slightly higher than the successful 40-bit fpzip and $A P A X 4 \mathrm{X}$ runs. With the $A P A X$ compressor, Miranda successfully ran with compression rates of $5.3 \mathrm{X}$.

A pF3D run with 32-bit fpzip (average compresion ratio 3.66) had low errors while a 24-bit fpzip run (average compression ratio 6.57) failed. The pF3D simulations using APAX passed the physics criteria at compression ratios up to $6 \mathrm{X}$. These results show that the onset of failure occurs across a fairly narrow range of compression ratios and can depend on interactions between the compressor and the code's numerics.

Lossy compression is a viable approach to reducing the impact of limited disk bandwidth for all three codes. The much more frequent compression necessary if arrays are decompressed each time they are loaded into cache is also acceptable if some caution is used in the requested compression level. Software compression takes longer than it does to transfer the uncompressed data between memory and cache on Intel Sandy Bridge systems. "Memory compression" software will hurt performance on current systems, but it will permit larger simulations to be run on a given number of nodes. Hardware compression schemes may be required to make "memory compression" a generally useful approach. For checkpointing, file formats such as HDF5 already provide support for lossless compression, and APAX is available as a compression filter for HDF5. The flexibility of libraries make it straightforward to also add support for other lossy compression schemes.

\section{Acknowledgment}

This document was released as LLNL-PROC-635789. This work was performed under the auspices of the U.S. Department of Energy by Lawrence Livermore National Laboratory under Contract DE-AC52-07NA27344. Some of this material is based upon work supported by the Defense Advanced Research Projects Agency under its Agreement No. HR0011-07-9-0001.

\section{REFERENCES}

[1] S. Moore, "Multicore is bad news for supercomputers," IEEE Spectrum, vol. 45, no. 11, 2008.

[2] R. Murphy, "On the effects of memory latency and bandwidth on supercomputer application performance," IEEE International Symposium on Workload Characterization, pp. 34-43, 2007.

[3] R. Chartrand, "Nonconvex compressive sensing and reconstruction of gradient-sparse images: Random vs. tomographic Fourier sampling," in IEEE International Conference on Image Processing, October 2008, pp. 2642-2627.
[4] S. Gleichman and Y. Eldar, "Blind compressed sensing," IEEE Transactions on Information Theory, vol. 57, no. 10, pp. 6958-6975, October 2011.

[5] E. Ozturk, O. Kucar, and G. Atkin, "Waveform encoding of binary signals using a wavelet and its Hilbert transform," IEEE International Conference on Acoustics, Speech, and Signal Processing, vol. 5, pp. 2641-2644, June 2000.

[6] D. A. Wright, "ADPCM coding and decoding techniques for personal communication systems," US Patent 5615 222, Mar., 1997.

[7] P. Lindstrom and M. Isenburg, "Fast and efficient compression of floating-point data," IEEE Transactions on Visualization and Computer Graphics, vol. 12, no. 5, pp. 1245-1250, 2006.

[8] M. Burtscher and P. Ratanaworabhan, "High throughput compression of double-precision floating-point data," in Data Compression Conference, March 2007, pp. 293-302.

[9] N. Huebbe and J. Kunkel, "Reducing the HPC-datastorage footprint with MAFISC multidimensional adaptive filtering improved scientific data compression," Computer Science Research and Development Journal, vol. 28, no. 2-3, pp. 231-239, May 2012.

[10] E. Schendel, Y. Jin, N. Shah, J. Chen, C. S. Chang, S.-H. Ku, S. Ethier, S. Klasky, R. Latham, R. Ross, and N. Samatova, "ISOBAR preconditioner for effective and high-throughput lossless data compression," in IEEE International Conference on Data Engineering, 2012, pp. 138-149.

[11] S. Muraki, "Approximation and rendering of volume data using wavelet transforms," in Proceedings of the $3 r d$ conference on Visualization '92, ser. VIS '92. Los Alamitos, CA, USA: IEEE Computer Society Press, 1992, pp. 21-28. [Online]. Available: http://dl.acm.org/citation.cfm?id=949685.949694

[12] _ _ "Multiscale volume representation by a dog wavelet," Visualization and Computer Graphics, IEEE Transactions on, vol. 1, no. 2, pp. 109-116, 1995.

[13] J. Woodring, S. Mniszewski, C. Brislawn, D. DeMarle, and J. Ahrens, "Revisiting wavelet compression for large-scale climate data using JPEG 2000 and ensuring data precision," in IEEE Large Data Analysis and Visualization, 2011, pp. 31-38.

[14] S. Lakshminarasimhan, N. Shah, S. Ethier, S. Klasky, R. Latham, R. Ross, and N. F. Samatova, "Compressing the incompressible with ISABELA: In-situ reduction of spatio-temporal data," in Euro-Par Parallel Processing, ser. Lecture Notes in Computer Science, 2011, pp. 366-379.

[15] H. Lehmann and B. Jung, "In-situ data compression for flow simulation in porous media," in Parallel $\&$ Distributed Processing Techniques 8 Applications, 2012.

[16] A. Wegener, "Adaptive compression and decompression of bandlimited signals," US Patent 7009 533, March, 2006. [Online]. Available: http:// www.patentlens.net/patentlens/patent/US_7009533/

[17] — , "Block floating point compression of signal data," US Patent 8301 803, October, 2012. [Online]. Available: http://www.patentlens.net/patentlens/ 
patent/US_8301803/

[18] P. Lindstrom, "fpzip version 1.0.1," 2008. [Online]. Available: https://computation.llnl.gov/casc/fpzip/

[19] N. Huebbe, A. Wegener, J. Kunkel, Y. Ling, and T. Ludwig, "Evaluating lossy compression on climate data," in International Supercomputing Conference, June 2013, pp. 343-356, iSC13 Proceedings.

[20] A. Wegener, N. Chandra, Y. Ling, R. Senzig, and R. Herfkens, "Effects of fixed-rate CT projection data compression on perceived and measured CT image quality," in SPIE Medical Imaging Proceedings, vol. 7627, Feb. 2010.

[21] A. Wegener, "Universal numerical encoder and profiler reduces computing memory wall with software, FPGA, and SoC implementations," in IEEE Data Compression Conference, Snowbird, UT (USA), March 2013, p. 528.

[22] L. Ibarria, P. Lindstrom, J. Rossignac, and A. Szymczak, "Out-of-core compression and decompression of large $n$-dimensional scalar fields," Computer Graphics Forum, vol. 22, no. 3, pp. 343-348, 2003.

[23] J. Keasler and R. Hornung, "Hydrodynamics Challenge Problem, Lawrence Livermore National Laboratory," Lawrence Livermore National Laboratory, Tech. Rep. LLNL-TR-490254, 2010.

[24] I. Karlin, A. Bhatele, B. L. Chamberlain, J. Cohen, Z. Devito, M. Gokhale, R. Haque, R. Hornung, J. Keasler, D. Laney, E. Luke, S. Lloyd, J. McGraw, R. Neely, D. Richards, M. Schulz, C. H. Still, F. Wang, and D. Wong, "LULESH programming model and performance ports overview," Lawrence Livermore National Laboratory, Tech. Rep. LLNL-TR-608824, December 2012.

[25] I. Karlin, A. Bhatele, J. Keasler, B. L. Chamberlain, J. Cohen, Z. DeVito, R. Haque, D. Laney, E. Luke, F. Wang, D. Richards, M. Schulz, and C. Still, "Exploring traditional and emerging parallel programming models using a proxy application," in IEEE International Parallel \& Distributed Processing Symposium, May 2013, to appear.

[26] M. L. Wilkins, Methods in Computational Physics. Academic Press, 1964.

[27] E. I. Moses, "Overview of the National Ignition Facility," Fusion Science and Technology, vol. 54, no. 2, pp. 361-366, 2008.

[28] E. I. Moses, R. N. Boyd, B. A. Remington, C. J. Keane, and R. Al-Ayat, "The National Ignition Facility: Ushering in a new age for high energy density science," Physics of Plasmas, vol. 16, no. 041006, pp. 1-13, 2009.

[29] C. H. Still, R. L. Berger, A. B. Langdon, D. E. Hinkel, L. J. Suter, and E. A. Williams, "Filamentation and forward Brillouin scatter of entire smoothed and aberrated laser beams," Physics of Plasmas, vol. 7, no. 5, pp. 2023-2032, 2000.

[30] R. L. Berger, B. F. Lasinski, A. B. Langdon, T. B. Kaiser, B. B. Afeyan, B. I. Cohen, C. H. Still, and E. A. Williams, "Influence of spatial and temporal laser beam smoothing on stimulated brillouin scattering in filamentary laser light," Phys. Rev. Lett., vol. 75, no. 6, pp. 1078-1081, Aug 1995.
[31] S. Langer, B. Still, T. Bremer, D. Hinkel, B. Langdon, J. A. Levine, and E. A. Williams, "Cielo full-system simulations of multi-beam laser-plasma interaction in NIF experiments," in Proceedings of the 53rd Cray User Group Meeting, 2011.

[32] A. W. Cook, W. H. Cabot, M. L. Welcome, P. L. Williams, B. J. Miller, B. R. de Supinski, and R. K. Yates, "Tera-scalable algorithms for variable-density elliptic hydrodynamics with spectral accuracy," in ACM/IEEE Conference on Supercomputing, 2005, p. 60.

[33] W. H. Cabot and A. W. Cook, "Reynolds number effects on Rayleigh-Taylor instability with possible implications for type-Ia supernovae," Nature Physics, vol. 2, pp. 562-568, 2006.

[34] A. W. Cook, W. Cabot, and P. L. Miller, "The mixing transition in Rayleigh-Taylor instability," Journal of Fluid Mechanics, vol. 511, pp. 333-362, 2004.

[35] E. Tasker, R. Brunino, N. Mitchell, D. Michielsen, S. Hopton, F. Pearce, G. Bryan, and T. Theuns, "A test suite for quantitative comparison of hydrodynamics codes in astrophysics," Monthly Notices of the Royal Astronomical Society, vol. 390, no. 3, pp. 1267-1281, 2008. 